\title{
Premiers travaux sur l'identification de différentes espèces de Trichostrongylus en Iran
}

\author{
par Esmaïl GHADIRIAN, Chamseddine MOFIDI et Hossein BIJAN
}

Faculté d'Hygiène et Institut de Recherches de la Santé publique de l'Université de Téhéran (Iran)

\section{Résumé}

I. - 20.732 Trichostrongylus furent récoltés chez 124 malades traités par l'Alcopar à Téhéran, Ispahan et au Khuzestan.

Six espèces différentes furent trouvées: $1 . T$. orientalis; 2. T. colubriformis; 3. T. probolurus; 4. T. vitrinus; 5 . T. axei; 6. T. skrjabini.

II. - Le taux d'infestation par Trichostrongylus dans le Nord de l'Iran est plus bas qu'ailleurs. Il atteint seulement 7,5\%.

Après le traitement par l'Alcopar, 4 espèces différentes furent trouvées ( $T$. colubriformis, $T$. vitrinus, $T$. axei, et $T$. orientalis). Le taux peu élevé de l'infection est probablement lié à la rareté de $T$. orientalis.

\section{Summary}

I. - 20.732 Trichostrongylus were isolated from 124 patients treated with Alcopar in Teheran, Ispahan and the Khuzestan.

Six differents species were found: 1. T. orientalis; $2 . T$. colubriformis; 3 . T. probolurus; 4. T. vitrinus; 5 . T. axei; 6. T. skrjabini.

II. - The rate of infection by Trichostrongylus in the North of Iran is lower than elsewhere. It reaches only $7,5 \%$.

After treatment with Alcopar 4 different species were found: (T. colubriformis, $T$. vitrinus, $T$. axei and $T$. orientalis). The rarety of $T$. orientalis is possibly responsible for the lower rate of infection.

Remerciements. - Nous tenons à remercier, tout particulièrement, le Professeur A.-G. Chabaud, professeur au Muséum National d'Histoire Naturelle de Paris, ainsi que le $\mathrm{D}^{\mathrm{r}} \mathrm{R}$. Sabokbar, le $\mathrm{D}^{r}$ A. Nadim, le $\mathrm{D}^{\mathrm{r}}$ F. Arfaa, le $\mathrm{D}^{\mathrm{r}}$ A. Amini, le $\mathrm{D}^{\mathrm{r}}$ A. Sadighian et F. Vedad pour leur aide efficace 
PREMIERE PARTIE

\section{Etude des Trichostrongylus des régions de Téhéran, Ispahan et du Khuzistan}

\section{I. - Introduction.}

Le degré d'infestation par les Trichostrongylus dans ces régions est de $42 \%$ pour Téhéran, $69 \%$ pour Ispahan (centre de l'Iran) et de plus de $60 \%$ pour le Khuzistan (près du Golfe persique). Téhéran et Ispahan jouissent d'un climat comparable. Le climat de Khuzistan est plus chaud et humide.

- Nous avons décidé de faire des recherches sur les différentes espèces de Trichostrongylus en raison du peu de renseignements que nous possédions à leur sujet, en ce qui concerne les adultes. Les travaux les plus récents ont été effectués à Ispahan par Biocca. Voici les conclusions de ses études dans cette région.

Etudes de Biocca: (2-3-4).

En 1959, Biocca a reçu quelques échantillons de matières fécales qu'on lui avait envoyé d'Ispahan. Après examen, il conclut que l'espèce humaine de Trichostrongylus en Iran est $T$. orientalis. Nous avons eu l'idée de poursuivre cette recherche par une méthode thérapeutique sur les individus porteurs de Trichostrongylus.

\section{II. - Méthodes et manipulations.}

Nous avons poursuivi nos recherches d'après le plan ci-dessous : les malades ont été choisis parmi les porteurs gravement infestés.

Parmi les médicaments anthelminthiques, nous avons retenu l'hydroxynaphtoate de Bephenium (Alcopar).

La veille du traitement, le malade est mis au régime sans légumes, ni fruits ; il boit seulement un verre de lait. Le lendemain, à jeun, il prend de l'Alcopar, puis on lui donne un laxatif salin. Les matières fécales sont recueillies dans un récipient contenant une solution de formol à $5 \%$. On recueille toutes les matières fécales émises dans les 24 heures, suivant la prise du médicament et du laxatif.

Procédés pour obtenir les Trichostrongylus.

Une quantité de matières fécales d'environ $100 \mathrm{~g}$ est mise dans un Bécher, et additionnée d'une certaine quantité d'eau chaude $\left(60^{\circ}\right.$ à $70^{\circ}$ centigrade) ; on délaye ensuite le contenu du récipient de façon à obtenir un mélange homogène ; ce mélange est filtré sur une passoire métallique dont les orifices ont un diamètre d'environ $1 \mathrm{~mm}$

* Ces études ont été financées par la Faculté d'Hygiène (Université de Téhéran) et l'Institut de Recherches de la Santé Publique (Organisation du Plan). 
et, après filtration, le résidu resté dans le filtre est placé dans un récipient cylindrique contenant de l'eau ; on laisse le mélange reposer pendant un certain temps (15 minutes) et on constate alors que le contenu du récipient s'est déposé en trois couches: une couche supérieure, constituée par des fibres végétales et quelques Trichostrongylus, une couche moyenne contenant uniquement du liquide et une couche inférieure comportant les substances lourdes et la majorité des Trichostrongylus. Pour obtenir les Trichostrongylus, il faut que les couches supérieure et surtout inférieure soient isolées et examinées séparément.

Les Trichostrongylus sont conservés dans une solution de formol à $5 \%$. L'expérience nous a montré que cette concentration évite la dégénérescence de ces vers.

Pour le montage, on utilise un mélange composé d'une quantité égale d'eau, d'alcool et de glycérine.

\section{Diagnostic :}

Pour identifier les différentes espèces de Trichostrongylus, nous avons choisi la méthode de Skrjabin (5).

\section{III. - Résultats.}

\section{1. - Téhéran.}

En 1964. pour la première fois en Iran, à partir des examens faits sur 157 sujets, nous avons trouvé 66 cas d'infestation (42\%). Pour obtenir facilement une quantité suffisante de Trichostrongylus, nous avons choisi 14 sujets fortement infestés. Après le traitement par l'Alcopar, nous avons obtenu, en tout, 996 Trichostrongylus adultes dont 713 étaient des femelles et 283, des mâles. Les résultats furent les suivants :

Parmi les 14 personnes infestées, 12 étaient porteuses de Trichostrongylus orientalis (fig. 1, A et B), mais les autres étaient à la fois porteuses de $T$. orientalis et de $T$. colubriformis. Il est à remarquer que, chez un seul porteur, on a trouvé 361 Trichostrongylus, dont 266 étaient des femelles et 95 des mâles.

Il faut signaler ici que chez les porteurs mixtes de Trichostrongylus orientalis et de $T$. colubriformis, le nombre total de $T$. orientalis était nettement supérieur au nombre de $T$. colubriformis.

\section{2. - Etudes faites dans la région d'Ispahan.}

Sur 579 examens de matières fécales effectués par la méthode de flottation, nous avons décelé 402 examens positifs, soit un pourcentage de $69 \%$. Dans ces 402 cas positifs, nous en avons choisi 60 , parmi les plus infestés, pour les soumettre à un traitement surveillé, et nous avons obtenu un total de 14.912 Trichostrongylus dont 5.229 étaient des mâles. 
Après vérification, sur 5.229 de Trichonstrongylus mâles, nous avons pu distinguer six espèces différentes :

I. Trichostrongylus orientalis (fig. 1, A. et B.).

II. Trichostrongylus colubriformis (fig. 2, C.).

III. Trichostrongylus probolurus (fig. 2, B.).
IV. Trichostrongylus vitrinus (fig. 3, A. et B.).

V. Trichostrongylus axsi (fig. 2, A.).

VI. Trichostrongylus skrjabini.

A.
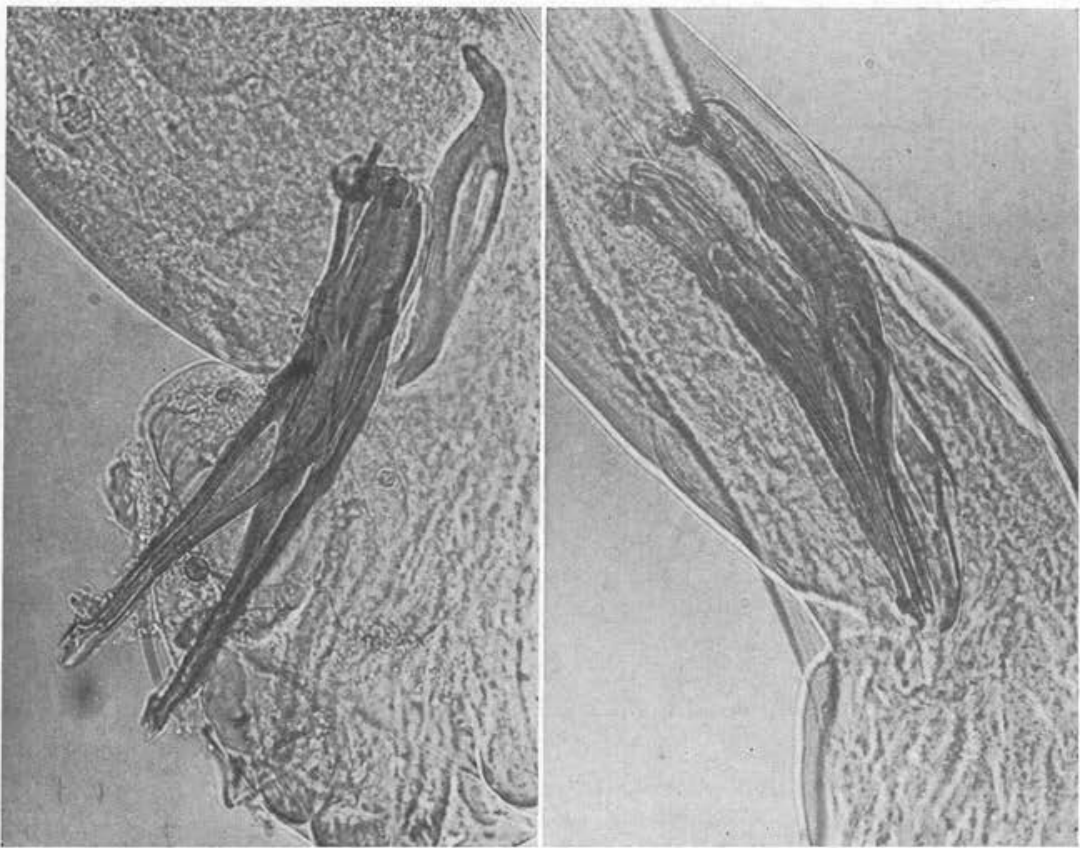

B.

FIG. 1. - Trichostrongylus orientalis

La distribution des 6 espèces de Trichostrongylus chez les 60 individus infestés était la suivante (tableau I) :

\section{Tableau I}

DISTRIBUTION DES 6 ESPÈCES DE Trichostrongylus CHEZ LES 60 SUJETS INFESTÉS D'IsPAHAN

\begin{tabular}{|c|c|c|c|c|c|c|c|}
\hline $\begin{array}{c}\text { Espèces de } \\
\text { Trichostrongylus }\end{array}$ & $T$. orientalis & $\begin{array}{l}\text { T.o.* } \\
\text { T.c.* }\end{array}$ & $\begin{array}{l}\text { T.o. } \\
\text { T.c. } \\
\text { T.v. }\end{array}$ & $\begin{array}{c}\text { T.o. } \\
\text { T.c. } \\
\text { T.a.* }\end{array}$ & $\begin{array}{l}\text { T.o. } \\
\text { T.a. }\end{array}$ & $\begin{array}{l}\text { T.o. } \\
\text { T.c. } \\
\text { T.p.* } \\
\text { T.a. } \\
\text { T.s.* }\end{array}$ & Total \\
\hline Nombre de sujets infestés & 30 & 19 & 6 & 2 & 2 & 1 & 60 \\
\hline
\end{tabular}

$*$ T.o. $=$ Trichostrongylus orientalis. ${ }^{*} T . c .=T$. colubriformis. ${ }^{*} T . p .=1$. probolurus, $* T . v .=T$. vitrinus. ${ }^{*} T . a .=T$. axei. $* T . s .=T$. skrjabini. 

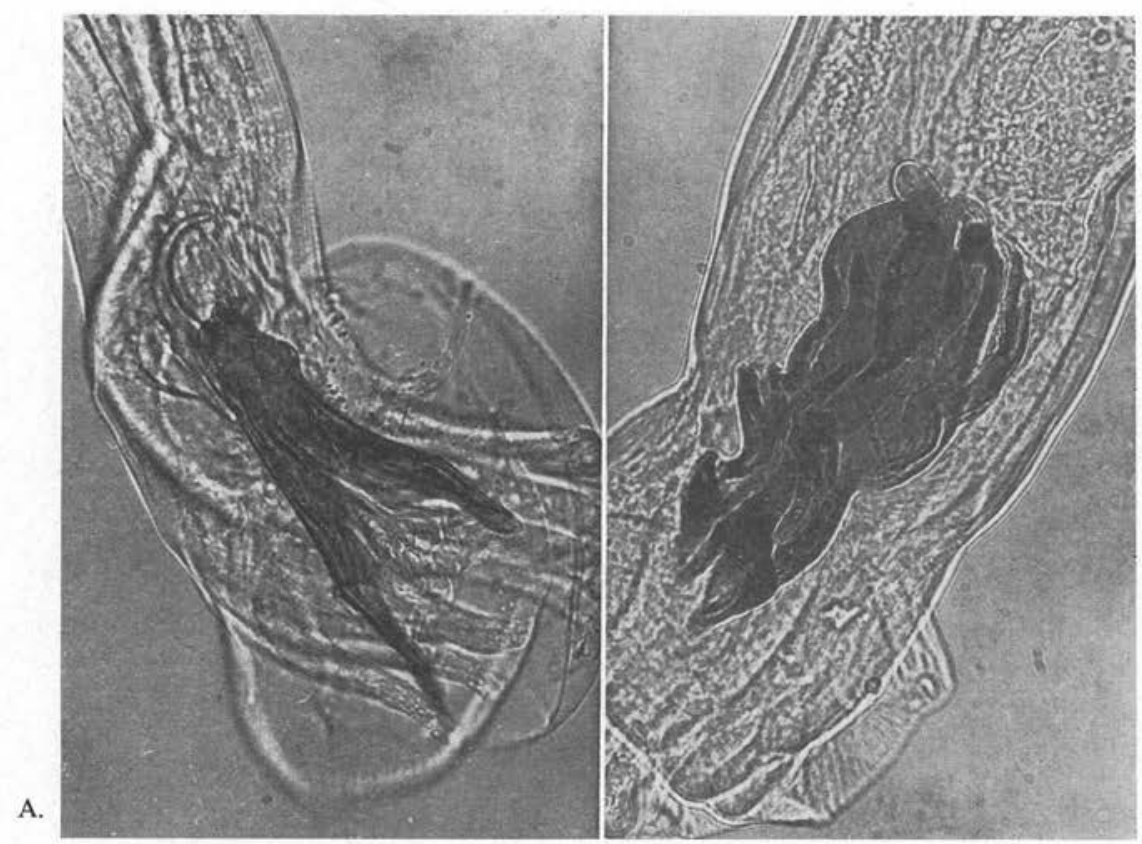

B.

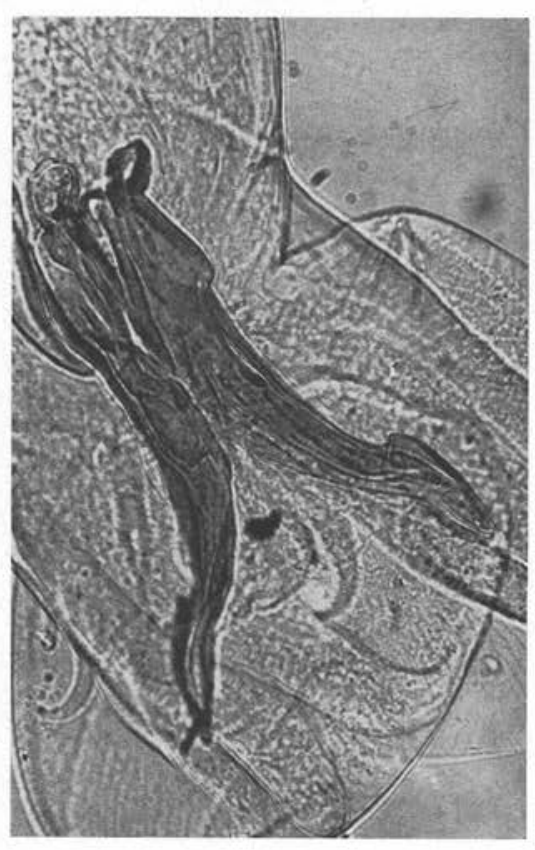

Fig. 2. - A: Trichostrongylus axei; B : $T$. probolurus; $-\mathrm{C}: T$. colubriformis. C. 
A.
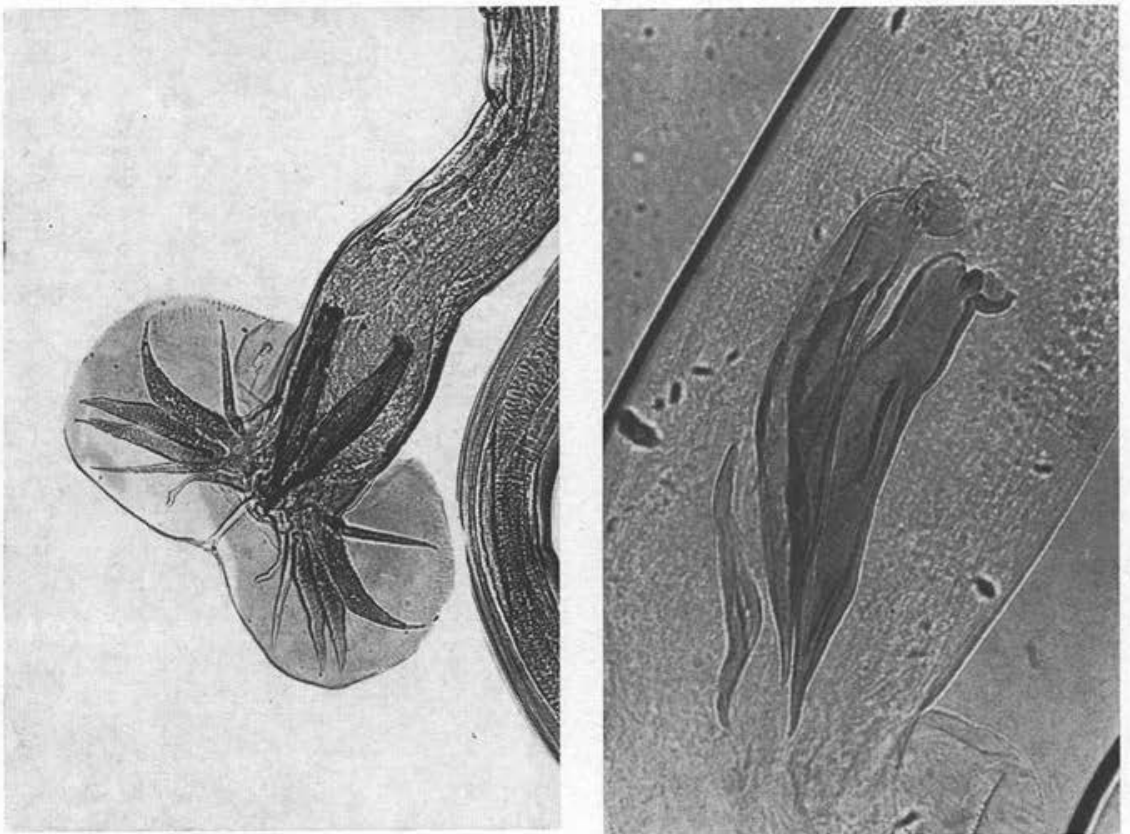

B.

FIG. 3. - Trichostrongylus vitrinus

Le nombre maximum de Trichostrongylus qu'on a pu trouver chez un seul sujet infesté était de 3.196, parmi lesquels, 2.643 étaient des femelles et le reste des mâles.

Ces 3.196 Trichostrongylus se répartissaient en cinq espèces:

I. T. orientalis. II. T. colubriformis. III. T. probolurus. IV. T. axei, V, T. skrjabini.

\section{3. - Au Khuzistan.}

Après le traitement d'une cinquantaine de sujets infestés, nous avons isolé 4.824 Trichostrongylus dont 2.827 femelles et 1.997 mâles. Ils appartenaient à 3 espèces différentes.

$T$. orientalis, $T$. colubriformis et $T$. vitrinus; mais le nombre des porteurs de Trichostrongylus orientalis était nettement supérieur à celui des porteurs de $T$. colubriformis et de $T$. vitrinus (tableau $\mathrm{n}^{\circ} \mathrm{II}$ ). 
Tableau II

DISTRIBUTION DES DIFFÉRENTES ESPÈCES DE Trichostrongylus CHEZ 50 sUJETS INFESTÉS aU KhuZistan

\begin{tabular}{|c|c|c|c|}
\hline Espèces & \multicolumn{2}{|c|}{$\begin{array}{c}\text { Nombre de Trichostrongylus } \\
\text { mâles }\end{array}$} & $\begin{array}{c}\text { Nombre de sujets } \\
\text { infestés }\end{array}$ \\
\hline$T$. orientalis $\ldots \ldots \ldots \ldots \ldots$ & 1.708 & & 25 \\
\hline T. colubriformis $\ldots \ldots \ldots \ldots$ & 25 & & 1 \\
\hline $\begin{array}{l}T . \text { orientalis } \ldots \ldots \ldots \ldots \ldots \ldots \\
T . \text { colubriformis } \ldots \ldots \ldots \ldots \ldots\end{array}$ & 252 & $\begin{array}{l}141 \text { T.o. } \\
111 \text { T.c. }\end{array}$ & 23 \\
\hline $\begin{array}{l}T . \text { orientalis } \ldots \ldots \ldots \ldots \ldots \\
T \text {. colubriformis } \ldots \ldots \ldots \ldots \\
T . \text { vitrinus } \ldots \ldots \ldots \ldots \ldots\end{array}$ & 12 & $\begin{array}{ll}5 & T . o . \\
4 & T . c . \\
3 & T . v .\end{array}$ & 1 \\
\hline Total $\ldots \ldots \ldots \ldots \ldots \ldots$ & 1.997 & & 50 \\
\hline
\end{tabular}

\section{Conclusion}

Après le traitement par l'Alcopar de 124 personnes infestées à Téhéran, à Ispahan et au Khuzestan, nous avons pu isoler 20.732 Trichostrongylus. Après identifications, nous avons distingué les six espèces ci-dessous.

I. $T$. orientalis. II. $T$. collubriformis. III. $T$. probolurus. IV. $T$. vitrinus. V. $T$. axei. VI. T. skrjabini.

Il ressort, de notre étude, que l'infestation par Trichostrongylus orientalis est la plus fréquente, puis, viennent, par ordre décroissant, $T$. colubriformis, $T$. vitrinus, $T$. axei, et, enfin, $T$. probolurus et $T$. skrjabini. A l'exception de $T$. orientalis, les autres espèces sont toujours associées. (Une fois seulement, nous avons isolé $T$. colubriformis comme seule espèce infestante chez un individu).

Identification des Trichostrongylus à Téhéran, Ispahan et au Khuzistan.

I. à Téhéran: $T$. orientalis et $T$. colubriformis.

II. à Ispahan : T. orientalis, $T$. colubriformis,

T. probolurus, T. vitrinus, T. axei et T. skrjabini.

III. au Khuzistan: T. orientalis, $T$. colubriformis et T. vitrinus.

Il faut, toutefois, observer que les renseignements apportés n'ont pas toujours été très complets et que les recherches sur les différentes espèces et la distribution de Trichostrongylus dans les autres régions de l'Iran se poursuivent encore actuellement. 


\section{DEUXIEME PARTIE}

\section{Etude sur les Trichostrongylus du Nord de l'Iran (rivages de la Mer Caspienne).}

\section{I. - Introduction.}

Géographiquement, le nord de l'Iran est séparé du reste du pays par les hautes chaînes montagneuses de l'Albourz. Cette situation lui confère un climat tout à fait différent des autres régions, de type méditerranéen. Les forêts y occupent une surface importante. La population locale se consacre à la culture du riz, du blé, du thé et du cotton, et à l'élevage des bovidés et des moutons.

Dans cette région, le degré d'infestation par les Trichostrongylus est nettement inférieur à ceux des régions de Téhéran, d'Ispahan et du Khuzistan que nous avons étudiés dans la première partie de ce travail.

D'après les enquêtes effectuées par le Centre de Recherches de la Santé publique à Chahsavar sur 1.706 sujets, le pourcentage d'infestation par Trichostrongylus ne s'élevait qu'à 7,5\%. L'intensité de l'infestation était également très faible. Par contre, il faut souligner que l'ankylostomiase est très répandue dans cette région.

\section{II. - Méthodes.}

Parmi les différents procédés permettant de mettre en évidence les Trichostrongylus adultes, nous avons choisi les méthodes thérapeutiques utilisant l'Alcopar et les techniques déjà décrites dans la première partie de ce travail.

\section{Choix des malades:}

Nous nous sommes efforcés de choisir nos sujets infestés parmi des individus originaires du nord et qui n'avaient pas quitté cette région, ceci afin de préciser les différentes espèces de Trichostrongylus qui peuvent se rencontrer au bord de la mer Caspienne. Ces sujets étaient tous des ruraux, vivant de la culture et de l'élevage.

\section{III. - Résultats.}

Trente et un sujets infestés provenant des villages voisins de Chahsavar étaient traités par l'Alcopar. Nous avons obtenu, en tout 559 Trichostrongylus dont 383 femelles et 176 mâles. Seuls, ces derniers nous ont servi à préciser les espèces en cause. Nous en avons trouvé quatre espèces dont la répartition est indiquée dans le tableau $\mathrm{n}^{\circ} 1$.

En tenant compte du fait que chez des 8 des 31 sujets étudiés, nous n'avons pu trouver que des Trichostrongylus femelles, le nombre d'individus ayant servi à étudier la répartition des 4 espèces se trouve ramené à 23 , seuls, les Trichostrongylus mâles permettant de bien préciser l'espèce en cause. Cette nouvelle répartition figure sur le tableau $\mathrm{n}^{\circ}$ II. 


\section{IV. - Discussion.}

Comme nous l'avons déjà souligné au début de cet article, le pourcentage et lintensité de l'infestation par les Trichostrongylus sont beaucoup plus faibles dans le nord de l'Iran que dans les autres régions.

Nous ne pouvons fournir une explication claire de cette différence. Un fait est certain: $T$. orientalis, espèce la plus fréquemment rencontrée dans les régions de Téhéran, d'Ispahan et au Khuzistan est très rare dans le Nord. Comme on peut le voir sur le tableau $\mathrm{n}^{\circ} \mathrm{I}$, sur 176 Trichostrongylus mâles isolés, il n'y avait que $2 T$. orientalis, soit un pourcentage inférieur à $2 \%$. Chez les animaux du Nord de l'Iran (moutons, chèvres et vaches), nous avons pu isoler 3 espèces de Trichostrongylus : T. vitrinus (la plus fréquente), $T$. colubriformis et $T$. axei, mais là encore, l'absence de $T$. orientalis est remarquable. Nous ne l'avons trouvé qu'une fois sur un anima! sauvage: le porcépic. Dans la région d'Ispahan, nous avons trouvé $T$. orientalis chez les moutons.

Cette rareté du $T$. orientalis dans le nord de l'Iran alors qu'il est très fréquent dans d'autres régions explique, peut-être, la moindre infestation de cette région. Il reste à découvrir pourquoi $T$. orientalis est beaucoup plus rare dans le Nord : ceci fera l'objet d'un prochain travail.

\section{Tableau I}

ESPÈCES DE Trichostrongylus TROUVÉES DANS LE NORD DE L'IRAN

\begin{tabular}{|c|c|c|c|c|c|}
\hline Espèces de Trichostrongylus .. & $\begin{array}{l}\text { T. colu- } \\
\text { briformis }\end{array}$ & $\begin{array}{l}\text { T. vitri- } \\
\text { nus }\end{array}$ & T. axei & $\begin{array}{l}\text { T. orien- } \\
\text { talis }\end{array}$ & Total \\
\hline $\begin{array}{l}\text { Nombre de Trichostrongylus } \\
\text { mâles } \ldots \ldots \ldots \ldots \ldots \ldots \ldots \ldots\end{array}$ & 97 & 41 & 36 & 2 & 176 \\
\hline
\end{tabular}

Tableau II

RÉPARTITION DES DIFFÉRENTES ESPÈCES DE Trichostrongylus CHEZ LES SUJETS INFESTÉS AU NORD DE L'IRAN

\begin{tabular}{|c|c|}
\hline Espèces de Trichostrongylus $\ldots \ldots \ldots \ldots \ldots \ldots$ & Nombre de sujets infestés \\
\hline$T$. colubriformis $\ldots \ldots \ldots \ldots \ldots \ldots \ldots \ldots \ldots$ & 6 \\
\hline T. vitrinus $\ldots \ldots \ldots \ldots \ldots \ldots \ldots \ldots \ldots \ldots \ldots \ldots \ldots \ldots \ldots \ldots$ & 3 \\
\hline$T$. colubriformis et $T$. vitrinus $\ldots \ldots \ldots \ldots \ldots$ & 2 \\
\hline T. colubriformis et $T$. axei $\ldots \ldots \ldots \ldots \ldots \ldots$ & 7 \\
\hline$T$. colubriformis, $T$. axei et $T$. vitrinus $\ldots \ldots \ldots$ & 4 \\
\hline$T$. orientalis et $T$. axei $\ldots \ldots \ldots \ldots \ldots \ldots \ldots$ & 1 \\
\hline 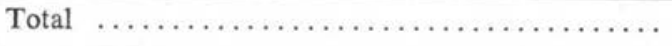 & 23 \\
\hline
\end{tabular}




\section{Conclusion}

- L'étude, sur l'infestation par Trichostrongylus dans le nord de l'Iran a permis de mettre en évidence la moindre intensité de cette infestation par rapport aux autres régions. En effet, celle-ci n'est que de $7,5 \%$.

- Par la thérapeutique à l'Alcopar, quatre espèces différentes ont pu être identifiées ( $T$. colubriformis, $T$. vitrinus, $T$. axei et $T$. orientalis).

- La rareté de $T$. orientalis est remarquable et explique peutêtre le degré moindre de l'infestation.

\section{Bibliographie}

1. Ansari (N.), 1950. - Parasitologie médicale, V. 2, p. 194. Faculté de Médecine de Téhéran.

2. BiocCa (E.), PAGG (L.) and ORECCHIA (P.), 1960. — Fürther studies on Trichostrongyliasis in Jewish Communities in Iran. Parassitologia, Rome 1960. Vol. 2, No. 3, p. 345-52.

3. - 1959. - Studies of intestinal helminthiasis in Jewish Communities in Iran. Parassitologia, Rome. Vol. 4, 1, 21-67.

4. -, PAGG (L.), 1959. - Infestazione sperimentale di ovis aries con larve di Trichostrongili umani provenienti dall' Iran. Parassitologia, 68-75.

5. SKRJABIN (K. J.), 1954. - Trichostrongylosis of animals and man. Essentials of Nematodology, V. 3. Academy of Sciences of the USSR Moscow.

6. Shahlapoor (A. A.), 1960. - T. axei in sheep and goats in Iran. First record, morphology. J. Helminth., 39 (213), p. 273-276.

7. - 1965. - A note on the identification of Skrjabinema ovis and Trichostrongylus spp. in sheep and goat in Iran. J. of. Helminth., XXXIX parts, 213, 1965.

8. Stewart (I. S.), 1949. - Human infestation with Trichostrongylus in South Persia. Brit. Med. J., 1949, I, 7378.

9. Watson (J. M.), 1960. - Medical Helminthology: Trichostrongylidae, p. 153, London, Bailliere Tindall and Cox. 